\title{
Square-Root Dynamics of a SIR-Model in Fractional Order
}

\author{
Young Il Seo ${ }^{1}$, Anwar Zeb ${ }^{2}$, Gul Zaman², Il Hyo Jung ${ }^{3}$ \\ ${ }^{1}$ National Fisheries Research and Development Institute, Busan, South Korea \\ ${ }^{2}$ Department of Mathematics, University of Malakand, Chakdara, Pakistan \\ ${ }^{3}$ Department of Mathematics, Pusan National University, Busan, South Korea \\ Email:*gzaman@uom.edu.pk
}

Received September 12, 2012; revised October 11, 2012; accepted October 18, 2012

\begin{abstract}
In this paper, we consider an SIR-model for which the interaction term is the square root of the susceptible and infected individuals in the form of fractional order differential equations. First the non-negative solution of the model in fractional order is presented. Then the local stability analysis of the model in fractional order is discussed. Finally, the general solutions are presented and a discrete-time finite difference scheme is constructed using the nonstandard finite difference (NSFD) method. A comparative study of the classical Runge-Kutta method and ODE45 is presented in the case of integer order derivatives. The solutions obtained are presented graphically.
\end{abstract}

Keywords: Mathematical Model; Square Root Dynamics; Fractional Derivative; Non-Standard Finite Difference Scheme; Numerical Analysis

\section{Introduction}

Mathematical epidemiology plays an important role in our society. Epidemic models to represent the interaction of different individuals by linear and nonlinear incidence have been discussed by many authors [1-3]. Literature of SIR diseases transmission model is quite large see [4-6], where $S$ represents the number of individuals that are susceptible to infection, $I$ represents the number of individuals that are infectious and $R$ denotes the number of individuals that have recovered. The SIR epidemic models are used in epidemiology to compute the amount of susceptible, infected and recovered people in a population. These models are also used to explain the dynamics of people in a community who need medical attention during an epidemic. However, it is important to note that these epidemic models do not work with all diseases. For the SIR model to be appropriate, once a person has recovered from the disease, they would receive lifelong immunity. But if a person was infected but is not infectious then someone need to modify the SIR epidemic model by including exposed class. The mathematical representation of SIR epidemic model consisting of three coupled ordinary equations which represents the dynamics of susceptible, infected and recovered individuals, respectively is given by

*Corresponding author.

$$
\begin{aligned}
& \frac{\mathrm{d} S}{\mathrm{~d} t}=\lambda-\beta f(S, I)-\mu S, \\
& \frac{\mathrm{d} I}{\mathrm{~d} t}=\beta f(S, I)-(\mu+\gamma) I, \\
& \frac{\mathrm{d} R}{\mathrm{~d} t}=\gamma I-\mu R,
\end{aligned}
$$

where $\lambda$ is the constant birth rate, $\mu$ is the natural death rate, $\gamma$ is the fraction of infected individuals who leave the infected class per unit time, $\beta$ is the rate of production of new infected individuals, and $f(S, I)$ is a function relating the rate of conversion of the susceptible population to the infected population. Mickens [7] introduced first time the square root interaction term in the SIR-model is given by

$$
\begin{aligned}
\frac{\mathrm{d} S(t)}{\mathrm{d} t} & =\lambda-\beta \sqrt{S(t) I(t)}-\mu S(t), \\
\frac{\mathrm{d} I(t)}{\mathrm{d} t} & =\beta \sqrt{S(t) I(t)}-(\mu+\gamma) I(t), \\
\frac{\mathrm{d} R(t)}{\mathrm{d} t} & =\gamma I(t)-\mu R(t),
\end{aligned}
$$

With

$$
S(0)=S_{0}, I(0)=I_{0}, R(0)=R_{0} .
$$

The total population is $N(t)=S(t)+I(t)+R(t)$. So we obtain by adding all equations of above system 


$$
\frac{\mathrm{d} N(t)}{\mathrm{d} t}=\lambda-\mu N(t) .
$$

Differential equations of fractional order have been the focus of many studies due to their frequent appearance in different applications in fluid mechanics, biology, physics, epidemiology and engineering. Recently, a large amount of literatures developed concerning the application of fractional differential equations in nonlinear dynamics [8-10]. The differential equations with fractional order have recently proved to be valuable tools to the modeling of many physical phenomena. This is because of the fact that the realistic modeling of a physical phenomenon does not depend only on the instant time, but also on the history of the previous time which can also be successfully achieved by using fractional calculus. The reason of using fractional order differential equations is that fractional order derivatives are naturally related to systems with memory which exists in most biological systems. Also they are closely related to fractals which are abundant in biological systems. As fractional calculus is the generalization of the ordinary differentiation and integration to non-integer and complex order. Also because of fractional order derivatives many authors established new models in different fields. In this paper, we consider a square root interaction in the SIR-model presented by Mickens [7] in fractional order. First we show the positive solution of square root interaction in the SIR epidemic model in fractional order. Then, we show the local stability of the epidemic model with fractional order. Finally, we compare our numerical results with nonstandard numerical method and fourth order Runge-Kutta method.

This paper is organized as: In Section 2, we present formulation of the model with some basic definitions and notations related to this work. In Section 3, we show the non-negative solution and uniqueness of the model. In Section 4, the local stability of the model is presented. In Section 5, the numerical simulations are presented graphically. Finally, we give conclusion.

\section{Formulation of Model with Preliminaries}

In this section, we present the SIR-model for which the interaction term is the square root of the susceptible and infected individuals in the form of fractional order differential equations. The new system is described by the following set of fractional order differential equations:

$$
\begin{aligned}
& D_{t}^{\alpha} S(t)=\lambda-\beta \sqrt{S(t) I(t)}-\mu S(t), \\
& D_{t}^{\alpha} I(t)=\beta \sqrt{S(t) I(t)}-(\mu+\gamma) I(t), \\
& D_{t}^{\alpha} R(t)=\gamma I(t)-\mu R(t), \\
& D_{t}^{\alpha} N(t)=\lambda-\mu N(t) .
\end{aligned}
$$

Here $D_{t}^{\alpha}$ is fractional derivative in the Caputo sense and $\alpha$ is a parameter describing the order of the fractional time-derivative with $0<\alpha<1$. For $\alpha=1$ the system will be reduced to ordinary differential equations. This kind of fractional differential equations is the generalizations of ordinary differential equations. Now we give some basic definitions related to this work and can be found in fractional calculus see for example [11-15].

Definition 1 A function $f(x)(x>0)$ is said to be in the space $C_{\alpha}(\alpha \in R)$ if it can be written as $f(x)=x^{p} f_{1}(x)$ for some $p>\alpha$ where $f_{1}(x)$ is con-tinuous in $[0, \infty)$, and it is said to be in the space $C_{\alpha}^{m}$ if $f^{(m)} \in C_{\alpha}, m \in N$.

Definition 2 The Riemann-Liouville integral operator of order $\alpha>0$ with $a \geq 0$ is defined as

$$
\begin{aligned}
& \left(J_{\alpha}^{\alpha} f\right)(x)=\frac{1}{\Gamma(\alpha)} \int_{a}^{x}(x-t)^{\alpha-1} f(t) \mathrm{d} t, x>a, \\
& \left(J_{a}^{0} f\right)(x)=f(x)
\end{aligned}
$$

Properties of the above operator can be found in [11].

Definition 3 For $f \in C_{\alpha}, \alpha, \beta>0, a \geq 0, c \in R$ and $\gamma>-1$, we have

$$
\begin{aligned}
& \left(J_{a}^{\alpha} J_{a}^{\beta} f\right)(x)=\left(J_{a}^{\beta} J_{a}^{\alpha} f\right)(x)=\left(J_{a}^{\alpha+\beta} f\right)(x) \\
& J_{a}^{\alpha} x^{\gamma}=\frac{x^{\gamma+\alpha}}{\Gamma(\alpha)} B_{\frac{x-a}{x}}(\alpha, \gamma+1)
\end{aligned}
$$

where $B_{\tau}(\alpha, \gamma+1)$ is the incomplete beta function which is defined as

$$
\begin{aligned}
& B_{\tau}(\alpha, \gamma+1)=\int_{0}^{\tau} t^{\alpha-1}(1-t)^{\gamma} \mathrm{d} t, \\
& J_{a}^{\alpha} \mathrm{e}^{c x}=\mathrm{e}^{a c}(x-a)^{\alpha} \sum_{k=0}^{\infty} \frac{[c(x-a)]^{k}}{\Gamma(\alpha+k+1)}
\end{aligned}
$$

The Riemann-Liouville derivative has certain disadvantages when trying to model real-world phenomena with fractional differential equations.

Definition 4 The Caputo fractional derivative of $f(x)$ of order $\alpha>0$ with $a \geq 0$ is defined as

$$
\left(D_{a}^{\alpha} f\right)(x)=\left(J_{a}^{m-\alpha} f^{(m)}\right)(x)=\frac{1}{\Gamma(m-\alpha)} \int_{a}^{x} \frac{f^{(m)}(t)}{(x-t)^{\alpha+1-m}} \mathrm{~d} t,
$$

for $m-1<\alpha \leq m, m \in N, x \geq a, f(x) \in C_{-1}^{m}$.

The fractional derivative was investigated by many authors, for $m-1<\alpha \leq m, f(x) \in C_{\alpha}^{m}$ and $\alpha \geq-1$, we have

$$
\left(J_{a}^{\alpha} D_{a}^{\alpha} f\right)(x)=J^{m} D^{m} f(x)=f(x)-\sum_{k=0}^{m-1} f^{(k)}(a) \frac{(x-a)^{k}}{k !} \text {. }
$$

The definition of fractional derivative involves an in- 
tegration which is non-local operator (as it is defined on an interval) so fractional derivative is a non-local operator. In other word, calculating time-fractional derivative of a function $f(t)$ at some $t=t_{1}$ time requires all the previous history, i.e. all $f(t)$ from $t=0$ to $t=t_{1}$.

\section{Non-Negative Solutions}

In this section, we show the positivity of the system. We first consider

$$
R_{+}^{4}=\left\{X \in R^{4}: X \geq 0\right\}
$$

and

$$
X(t)=(S(t), I(t), R(t), N(t))^{\mathrm{T}} .
$$

In order to prove the theorem about non-negative solutions, we need to state the following Lemma [9].

Lemma 3.1. (Generalized Mean Value Theorem) Let $f(x) \in C\lceil a, b\rceil$ and $D^{\alpha} f(x) \in C\lceil a, b\rceil$ for $0<\alpha \leq 1$. Then we have

$$
f(x)=f(a)+\frac{1}{\Gamma(\alpha)} D^{\alpha} f(\xi)(x-a)^{\xi},
$$

with $0 \leq \xi \leq x$, for all $x \in(a, b]$.

Remark 3.2. Suppose $f(x) \in C\lceil a, b\rceil$ and

$D^{\alpha} f(x) \in C[a, b]$ for $0<\alpha \leq 1$. It is clear from Lemma

3.1 that if $D^{\alpha} f(x)>0$ for all $x \in(0, b)$, then the function $f$ is non-decreasing, and if $D^{\alpha} f(x) \leq 0$ for all $x \in(0, b)$, then the function $f$ is non-increasing.

Theorem 3.3. There is a unique solution for the initial value problem given by (5)-(8), and the solution remains in $R_{+}^{4}$.

Proof. The existence and uniqueness of the solution of (5)-(8), in $(0, \infty)$ can be obtained from [5, Theorem 3.1 and Remark 3.2]. We need to show that the domain $R_{+}^{4}$ is positively invariant. Since

$$
\begin{aligned}
& \left.D_{t}^{\alpha} S(t)\right|_{S=0}=\lambda \geq 0,\left.D_{t}^{\alpha} I(t)\right|_{I=0}=0, \\
& \left.D_{t}^{\alpha} R(t)\right|_{R=0}=\gamma I(t) \geq 0,\left.D_{t}^{\alpha} N(t)\right|_{N=0}=\lambda \geq 0 .
\end{aligned}
$$

On each hyperplane bounding the non-negative orthant, the vector field points into $R_{+}^{4}$.

\section{Local Stability Analysis of Model}

The system of ODE's given by (1)-(3) has a unique non-trivial solution. By setting the right hand side of the Equations (1)-(3) equal to zero, we get

$$
\begin{aligned}
& S^{*}=\frac{\lambda(\mu+\gamma)}{\mu(\mu+\gamma)+\beta^{2}}, I^{*}=\left(\frac{\beta}{\mu+\gamma}\right)^{2} S^{*}, \\
& R^{*}=\left(\frac{\gamma}{\mu}\right)\left(\frac{\beta}{\mu+\gamma}\right)^{2} S^{*} .
\end{aligned}
$$

All the parameters are taken to be positive, then $S^{*}, I^{*}, R^{*}$ are positive. For the unique positive equilibria the Jacobian matrix at this fixed point is

$$
J\left(S^{*}, I^{*}, R^{*}\right)=\left(\begin{array}{ccc}
-a & -b & 0 \\
c & -b & 0 \\
0 & \gamma & -\mu
\end{array}\right)
$$

here

$$
a=\frac{2 \mu(\mu+\gamma)+\beta^{2}}{2(\mu+\gamma)}, b=\frac{\mu+\gamma}{2}, c=\frac{\beta^{2}}{2(\mu+\gamma)} .
$$

The eigen values $\left(\lambda_{1}, \lambda_{2}, \lambda_{3}\right)$ are given by

$$
\operatorname{det}\left[J\left(S^{*}, I^{*}, R^{*}\right)-\lambda I_{3}\right]=0 \text {, }
$$

where $I_{3}$ is the unit matrix of order $3 \times 3$. By evaluating this determinant we obtain the following equation

$$
(\lambda+\mu)\left(\lambda^{2}+(a+b) \lambda+a b+b c\right)=0 .
$$

It is clear that $\lambda_{1}=-\mu$ is negative. For others roots we can write

$$
\lambda^{2}+(a+b) \lambda+a b+b c=0 .
$$

Let the remaining roots of this equation are $\lambda_{2}$ and $\lambda_{3}$, that satisfying the following relations

$$
\lambda_{2} \lambda_{3}=a b+b c>0, \lambda_{2}+\lambda_{3}=-a b-b c<0 .
$$

From Equation (11) we conclude that:

1) If $\lambda_{2}$ and $\lambda_{3}$ are real, then both roots have same sign.

2) If $\lambda_{2}$ and $\lambda_{3}$ are real, then both roots are negative.

3) If $\lambda_{2}$ and $\lambda_{3}$ are complex, then $\lambda_{2}=\lambda_{3}$ and the real parts are negative.

4) Thus, all the eigenvalues are negative or have negative real parts, and hence we conclude that this fixed point is located at $\left(S^{*}, I^{*}, R^{*}\right)$ is locally stable.

\section{The NSFD Scheme}

In general, the non-standard finite difference rules, introduced by Mickens [7,16-19], do not lead to a discrete model for the unique solution of any dynamical system based on differential equations. First, we give the basic rules of nonstandard ordinary differential equations (ODEs) is given by

$$
y_{k}^{\prime}=f\left(t, y_{1}, y_{2}, \cdots, y_{m}\right), k=1,2, \cdots, m .
$$

where $f\left(t, y_{k}(t)\right)$ is the nonlinear term in the differential equation. Using the finite difference method we have

$$
\begin{aligned}
& y_{1}^{\prime}=\frac{y_{1, k+1}-y_{1, k}}{\phi_{k}(h)}, y_{2}^{\prime}=\frac{y_{2, k+1}-y_{2, k}}{\phi_{k}(h)}, \cdots, \\
& y_{m}^{\prime}=\frac{y_{m, k+1}-y_{m, k}}{\phi_{k}(h)}
\end{aligned}
$$


where $\phi_{k}$ is a function of the step size $h=\Delta t$. The function $\phi_{k}$ have the following properties:

$$
\phi_{k}(h)=h+o\left(h^{2}\right), \text { for } h \rightarrow 0 .
$$

Examples of functions $\phi_{k}(h)$ that satisfy (12) are $h$,

$$
\sin (h), \sinh (h), \mathrm{e}^{h}-1, \frac{1-\mathrm{e}^{-\lambda h}}{\lambda} .
$$

Non-linear terms can in general be replaced by nonlocal discrete representations, for example

$$
y^{2} \approx y_{k} y_{k+1}, y^{3} \approx\left(\frac{y_{k+1}+y_{k-1}}{2}\right) y_{k}^{2},
$$

here $h=\frac{T}{N}, t_{n}=n h, n=0,1, \cdots, \mathbf{N} \in \mathbf{Z}^{+}$.

The NSFD scheme for (1)-(4) system is shown as follows:

$$
\begin{aligned}
& \frac{S_{k+1}-S_{k}}{\phi}=\lambda-\beta \sqrt{S_{k+1} I_{k}}-\mu S_{k+1}, \\
& \frac{I_{k+1}-I_{k}}{\phi}=\beta \sqrt{S_{k+1} I_{k}}-(\mu+\gamma) I_{k+1}, \\
& \frac{R_{k+1}-R_{k}}{\phi}=\gamma I_{k+1}-\mu R_{k+1}, \\
& \frac{N_{k+1}-N_{k}}{\phi}=\lambda-\mu N_{k+1} .
\end{aligned}
$$

Here

$$
N_{k}=S_{k}+I_{k}+R_{k} .
$$

Now making the transformation of variables

$$
u_{k+1}=\sqrt{S_{k+1}},
$$

in the first equation of system (13), we obtain a quadratic equation for $u_{k+1}$,

$$
\begin{aligned}
& (1+\phi(\mu)) u_{k+1}^{2}+\left(\beta \phi \sqrt{I_{k}}\right) u_{k+1} \\
& -\left(S_{k}+\phi \lambda\right)=0 .
\end{aligned}
$$

Note that our interest is calculating $S_{k+1}$ which is based on the knowledge of $\left(\lambda, \mu, \beta, S_{k}, I_{k}\right)$, and then we used the transformation given in Equation (14). The solution for the quadratic Equation (15) is

$$
\begin{aligned}
& u_{k+1}=\left[\frac{1}{2(1+\phi(\mu))}\right] \\
& \cdot\left[-\left(\beta \phi \sqrt{I_{k}}\right)+\sqrt{\left(\beta \phi \sqrt{I_{k}}\right)^{2}+4(1+\mu)\left(S_{k}+\phi \lambda\right)}\right]
\end{aligned}
$$

Similarly, the remaining equations of the system (13) can be solved for the variables at the $(k+1)$ th time step:

$$
\begin{aligned}
& I_{k+1}=\left[\frac{1}{(1+\phi(\gamma+\mu))}\right]\left[\left(\beta \phi \sqrt{I_{k}}\right) u_{k+1}+I_{k}\right], \\
& R_{k+1}=\left[\frac{1}{(1+\phi(\mu))}\right]\left[\gamma \phi I_{k+1}+R_{k}\right], \\
& N_{k+1}=\left[\frac{1}{(1+\phi(\mu))}\right]\left[\lambda \phi+N_{k}\right] .
\end{aligned}
$$

\section{Numerical Method and Simulation}

In this section we find the numerical solutions. For numerical simulation, we use $\mu=0.04, \gamma=0.03, \beta=0.05$ and $\lambda=1$. For the effectiveness of the proposed algorithm which as an approximate tools for the solution of the nonlinear system of fractional differential Equations (1)-(4). Figures 1-4 show the approximate solutions obtained using ODE45 and classical RK4 method of $S(t)$, $I(t), R(t)$ and $N(t)$ when $\alpha=1$. Figures 5-8 show the

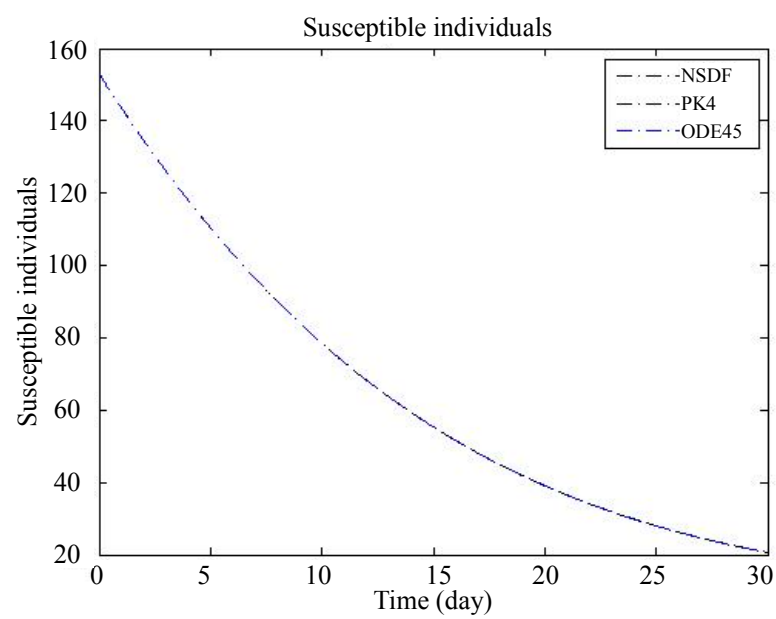

Figure 1. The plot represents the susceptible individuals.

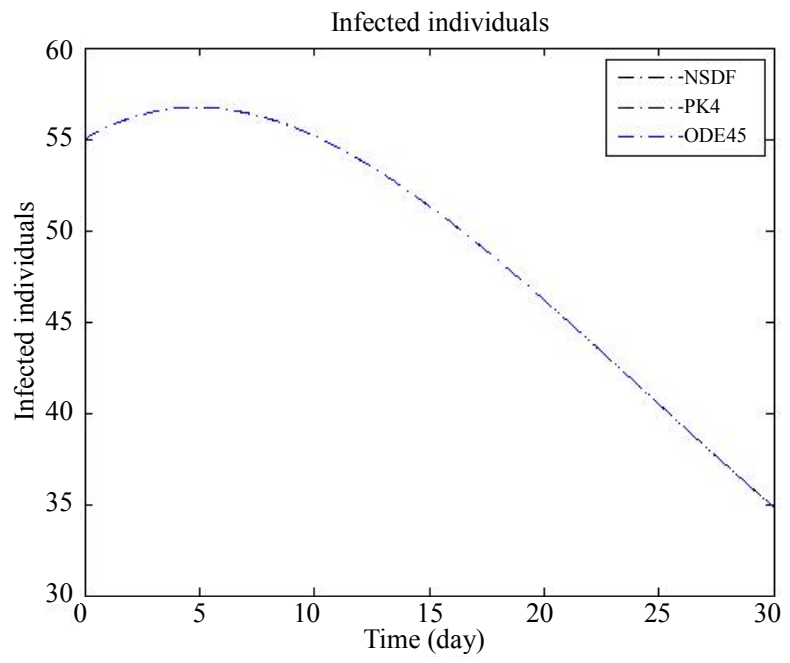

Figure 2. The plot represents the infected individuals. 


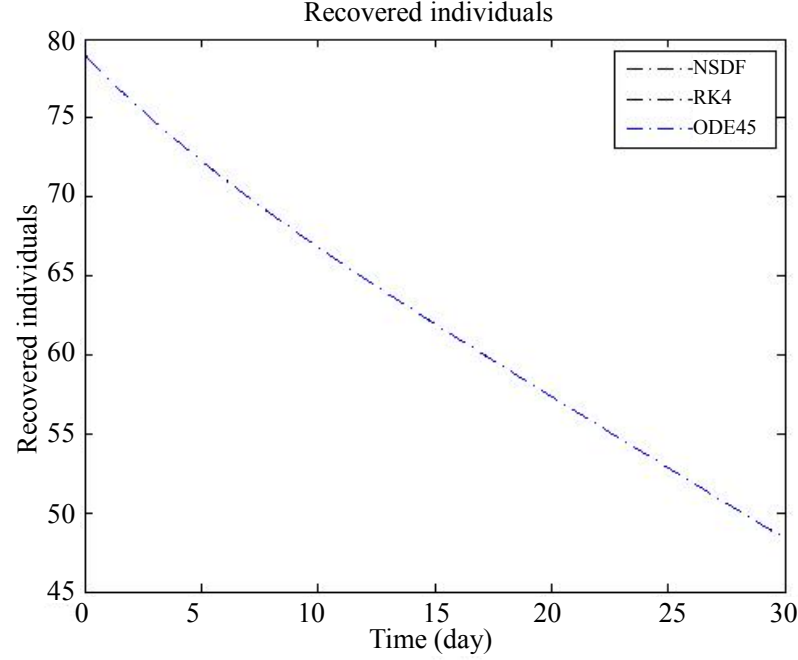

Figure 3. The plot represents the recovered individuals.

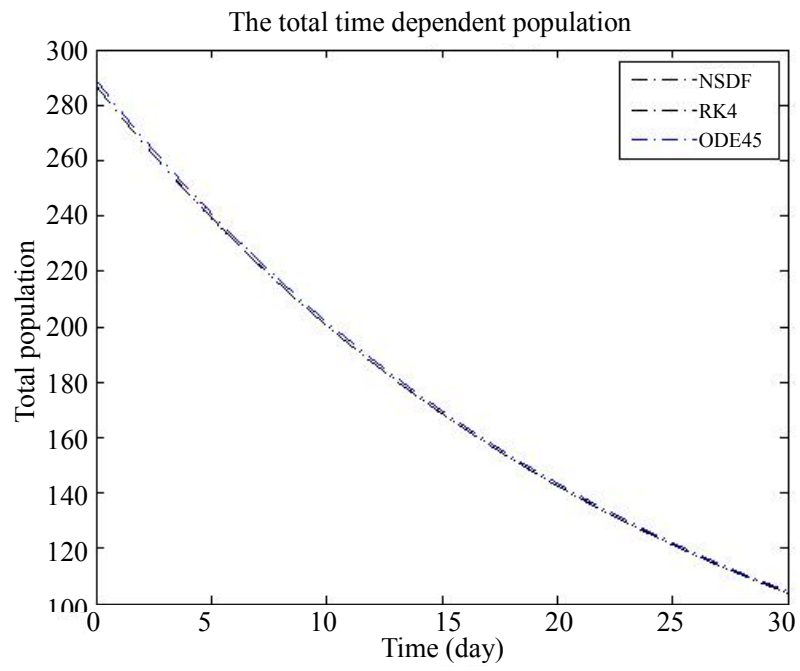

Figure 4. The plot represents the total population.

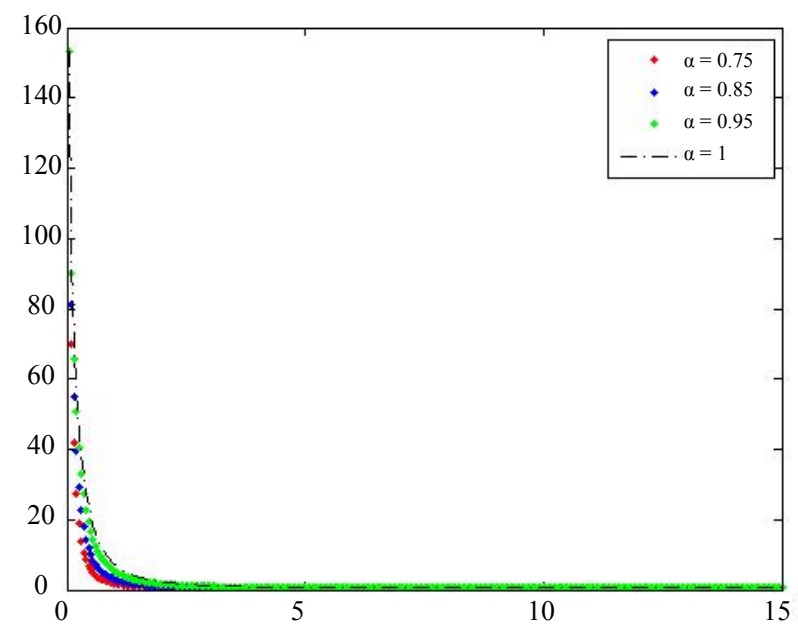

Figure 5. The plot represents the susceptible individuals for different values of $\alpha$.

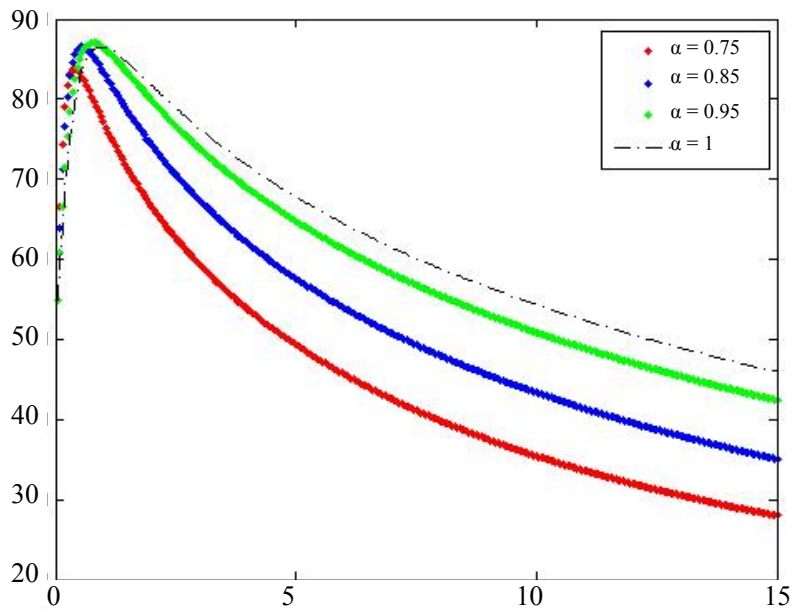

Figure 6. The plot represents the infected individuals for different values of $\alpha$.

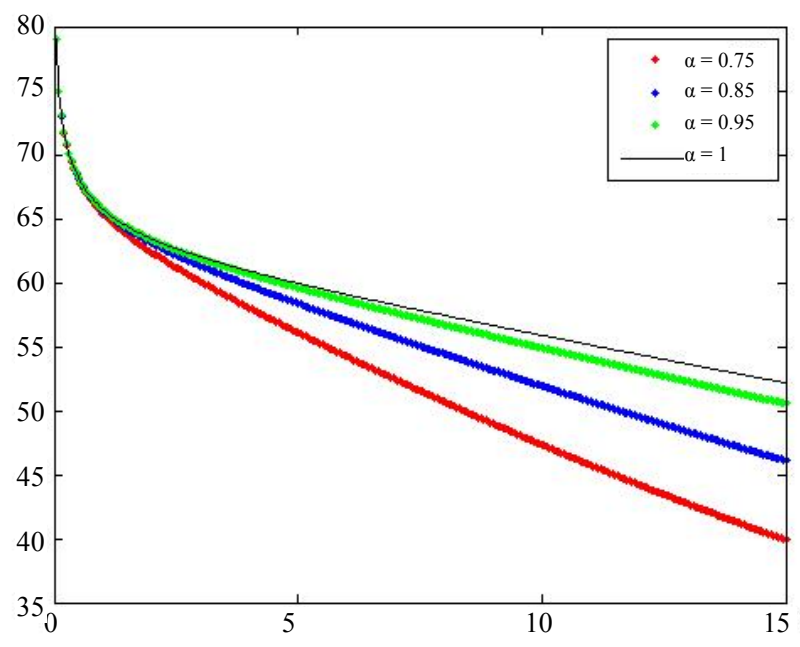

Figure 7. The plot represents the recovered individuals for different values of $\alpha$.

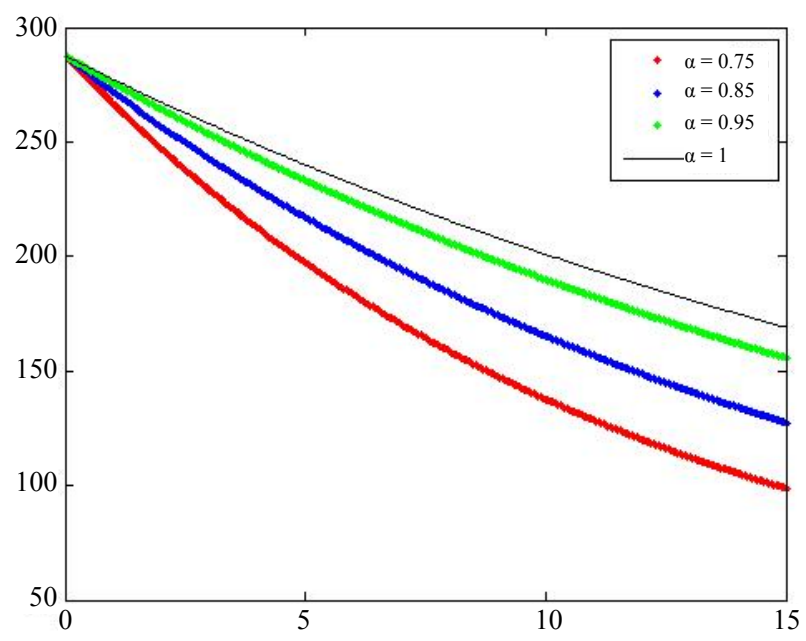

Figure 8. The plot represents the total population for different values of $\alpha$. 
approximate solutions of $S(t), I(t), R(t)$ and $N(t)$ for $\alpha=$ $0.75,0.85,0.95,1$.

\section{Conclusion}

In this paper, we introduced fractional derivatives in the SIR epidemic model with square root interaction of the susceptible and infected individuals. First the non-negative solution of the model in fractional order is presented. Then the local stability analysis of the model in fractional order is presented. Finally, the general solutions were also discussed and a discrete-time, finite difference scheme is constructed using the nonstandard finite difference (NSFD) method.

\section{Acknowledgements}

This study was founded by the National Fisheries Research and Development Institute (RP-2012-FR-040).

\section{REFERENCES}

[1] B. Adams and M. Boots, "The Influence of Immune Cross-Reaction on Phase Structure in Resonant Solutions of a Multi-Strain Seasonal SIR Model," Journal of Theoretical Biology, Vol. 248, No. 1, 2007, pp. 202-211. doi:10.1016/j.jtbi.2007.04.023

[2] R. M. Anderson and R. M. May, "Infectious Disease of Humans, Dynamics and Control," Oxford University Press, Oxford, 1991.

[3] C. Castillo-Garsow, G. Jordan-Salivia and A. Rodriguez Herrera, "Mathematical Models for Dynamics of Tobacco Use, Recovery and Relapse," Technical Report Series BU-1505-M, Cornell Uneversity, Ithaca, 2000.

[4] M. Choisy, J. F. Guezan and P. Rohani, "Dynamics of Infectious Diseases and Pulse Vaccination: Teasing Apart the Embedded Resonance Effects," Journal of Physics D, Vol. 223, No. 1, 2006, pp. 26-35. doi:10.1016/j.physd.2006.08.006

[5] M. G. Gomes, L. J. White and G. F. Medley, "Infection, Reinfection, and Vaccination under Suboptimal Immune Protection: Epidemiological Perspectives," Journal of Theoretical Biology, Vol. 228, No. 4, 2004, pp. 539-549. doi:10.1016/j.jtbi.2004.02.015

[6] G. Zaman and I. H. Jung, "Optimal Vaccination and Treatment in the SIR Epidemic Model," Proceedings of KSIAM, Vol. 3, No. 2, 2007, pp. 31-33.

[7] R. E. Mickens, "A SIR-Model with Square-Root Dynamics: An NSFD Scheme," Journal of Difference Equations and Applications, Vol. 16, No. 2-3, 2009, pp. 209-216.

[8] Z. M. Odibat and N. T. Shawafeh, "Generalized Taylor's Formula," Computers \& Mathematics with Applications, Vol. 186, No. 1, 2007, pp. 286-293. doi:10.1016/j.amc.2006.07.102

[9] W. Lin, "Global Existence Theory and Chaos Control of Fractional Differential Equations," Journal of Mathematical Analysis and Applications, Vol. 332, No. 1, 2007, pp. 709-726.

[10] L. Debnath, "Recent Applications of Fractional Calculus to Science and Engineering," International Journal of Mathematics and Mathematical Sciences, Vol. 2003, No. 54, 2003, pp. 3413-3442.

[11] I. Podlubny, "Fractional Differential Equations," Academic Presss, London, 1999.

[12] D. Matignon, "Stability Results for Fractional Differential Equations with Applications to Control Processing," Computational Engineering in System Application, Vol. 2 1996, p. 963.

[13] V. S. Erturk, Z. M. Odibait and S. Momani, "An approximate Solution of a Fractional Order Differential Equation Model of Human T-Cell Lymphotropic Virus I HTLV-I, Infection of CD4 T-Cells," Computers \& Mathematics with Applications, Vol. 62, No. 3, 2011, pp. 996-1002. doi:10.1016/j.camwa.2011.03.091

[14] S. Miller and B. Ross, "An Introduction to the Fractional Calculus and Fractional Differential Equations," Willey, New York, 1993.

[15] N. Ozalp and E. Demirci, "A Fractional Order SEIR Model with Vertical Transmission," Mathematical and Computer Modelling, Vol. 54, No. 1, 2011, pp. 1-6. doi:10.1016/j.mcm.2010.12.051

[16] R. E. Mickens, "Nonstandard Finite Difference Models of Differential Equations," World Scientific, Singapore, 1994.

[17] R. E. Mickens, "Calculation of Denominator Functions for NSFD Schemes for Differential Equations Satisfying a Positivity Condition," Numerical Methods for Partial Differential Equations, Vol. 23, No. 3, 2007, pp. 672-691. doi:10.1002/num.20198

[18] R. E. Mickens, "Numerical Integration of Population Models Satisfying Conservation Laws: NSFD Methods," Journal of Biological Dynamics, Vol. 1, No. 4, 2007, pp. 427-436. doi:10.1080/17513750701605598

[19] R. E. Mickens, R. Buckmire and K. McMurtry, "Numerical Studies of a Nonlinear Heat Equation with Square Root Reaction Term," Numerical Methods for Partial Differential Equations, Vol. 25, No. 3, 2009, pp. 598-609. doi:10.1002/num.20361 